\title{
A CHARACTERIZATION OF PRIMITIVE BASES
}

\author{
H. H. WICKE AND J. M. WORRELL, JR.
}

ABSTRACT. The concept of primitive base is characterized in terms of primitive sequences. A characterization of essentially $T_{1}$ spaces having bases of countable order is a corollary.

1. Introduction. The concept of primitive base is fundamentally related to spaces having bases of countable order (see Theorem 2.6). It therefore underlies in a basic way such important topological structures as developable spaces and metrizable spaces. The main purpose of this article is to characterize primitive base in terms of primitive sequences, i.e., by a certain structure defined with the use of a sequence of well-ordered open coverings. This result shows that spaces having primitive bases are prototypes of both spaces having $\theta$-bases (equivalently, quasi-developable spaces [2]) and of spaces having bases of countable order. The presence in such spaces of what we call a primitive sequence of basic type (see 4.2) permits the development of an extensive and harmonious theory [8]-[10], [12]-[14] wider in scope than the theories of quasi-developable spaces or of spaces having bases of countable order.

In $\$ 2$ we make some detailed comparisons. In $\$ 3$ we prove the main theorem. In $\$ 4$ we obtain a new characterization of spaces having bases of countable order. In $\$ 5$ we discuss completeness. We shall discuss elsewhere additional properties of spaces having primitive bases and a natural generalization to non-first-countable spaces arising from the main theorem.

Notation and terminology. Sequences such as $\left\langle H_{n}: n \in N\right\rangle$ will be denoted by $H$. Recall that a space $X$ is essentially $T_{1}$ [12] if and only if for all $x, y \in X, x \in\{\bar{y}\}$ implies $y \in\{\bar{x}\}$ (see [3] also). The letter $N$ denotes the set of positive integers. The notations $<$ and $\leq$ are used ambiguously to denote orderings whose domains and ranges are contextually clear.

Received by the editors April 10, 1974.

AMS (MOS) subject classifications (1970). Primary 54E99; Secondary 54E30, 54A99.

Key words and phrases. Primitive base, primitive sequence, $\theta$-base, base of countable order, quasi-developable space, developable space, completeness. 
2. Primitive base. We define primitive base and show that it generalizes base of countable order and $\theta$-base. Theorem 2.6 , stated without proof, gives a precise connection with essentially $T_{1}$ spaces having bases of countable order (see also 4.3).

2.1. Definition [11]. A topological space $X$ is said to have a primitive base if and only if there exists a sequence $W$ whose terms are well-ordered collections of open sets such that, for all $x \in X$, if $U$ is open and $x \in U$, then there exist integers $k$ and $n$ such that $x$ is in $n$ elements of $6_{k}$ and the $n$th such element is a subset of $U$.

2.2. Definition [12]. A topological space is said to have a $\theta$-base if and only if there exists a collection $B$ of open sets in $X$ such that $B=\bigcup_{n \in N} B_{n}$ and for each $x \in X$, if $U$ is open and $x \in U$, then there exists $n \in N$ such that $\left\{B \in B_{n}: x \in B\right\}$ is finite and some member of this set is a subset of $U$.

2.3. Theorem [2]. A topological space $X$ has a $\theta$-base if and only if it is quasi-developable, i.e., there exists a sequence $\mathcal{G}$ of collections of open subsets of $X$ such that for each $x \in X$ if $U$ is open and $x \in U$ there exists $n$ such that $x \in \operatorname{st}\left(x, \varrho_{n}\right) \subseteq U$.

2.4. Theorem. Every topological space which has a $\theta$-base, or is quasidevelopable, or is an essentially $T_{1}$ space having a base of countable order, has a primitive base.

Proof. The first two statements are clear from the definitions and 2.3. The last statement follows from 3.6 and 4.1 .

2.5. Example. Let $X$ denote the Michael line [4] and $\omega_{1}$ the space of countable ordinals with the order topology. Then the topological sum $X \oplus \omega_{1}$ and the topological product $X \times \omega_{1}$ are spaces which have primitive bases but which have neither a base of countable order nor a $\theta$-base.

Proof. The space $X$ has a base consisting of the irrational singletons and the collection of all balls $B(x, 1 / n)$ where $x$ is rational and $n \in N$. Let $\left\{x_{n}: n \in N\right\}$ be the range of a bijection between $N$ and the set of rational numbers. Let $B_{00}=\{\{y\}: y$ is irrational $\}$ and $\mathscr{B}_{n m}=\left\{B\left(x_{n}, 1 / m\right)\right\}$ for $n, m \in N$. The collection $\mathfrak{B}_{00} \cup \bigcup_{n, m \in N} \mathfrak{B}_{n m}$ is a $\theta$-base for $X$. The space $\omega_{1}$ has a base of countable order but is not developable [15 (in different terminology)]. That $\omega_{1}$ does not have a $\theta$-base follows from the result of [2] that a linearly ordered space is quasi-developable if and only if it is paracompact, since $\omega_{1}$ is not paracompact. The property of having a $\theta$-base is hereditary as is that of having a base of countable order [12]. Hence neither $X \oplus \omega_{1}$ nor $X \times \omega_{1}$ can have a $\theta$-base or a base of countable order. That $X \oplus \omega_{1}$ has a primitive 
base is clear from 2.4 and the definition of topological sum. That $X \times \omega_{1}$ has a primitive base follows from 2.4 and 3.9.

The following theorem, whose proof is made accessible by Theorem 3.6, will be proved elsewhere. For set of interior condensation see [5].

2.6. Theorem [11], [13]. A topological space is essentially $T_{1}$ and has a base of countable order if and only if it has a primitive base and closed sets are sets of interior condensation locally.

This result makes interesting contrast to the next two (known) theorems.

2.7. Theorem [12]. A topological space is developable if and only if it has a $\theta$-base and closed sets are $G_{\delta}$ sets.

A theorem equivalent in content to 2.7, in view of Theorem 2.3, is the next one, discovered by Bennett independently of 2.3 and 2.7.

2.8. Theorem [1]. A topological space is developable if and only if it is quasi-developable and closed sets are $G_{\delta}$ sets.

3. Primitive sequences and primitive bases. We first present some terminology of the theory of primitive sequences [6], and then characterize essentially $T_{1}$ spaces having primitive bases.

3.1. Definition. Let $(\mathcal{Z}, \leq)$ be a well-ordered collection of sets. For each $W \in \mathscr{Z}$ let $p(W, \mathscr{Z})$ denote $\left\{x \in W: W^{\prime} \in \mathcal{Z}\right.$ and $W^{\prime}<W$ implies $\left.x \notin W^{\prime}\right\}$. The set $p(W, \mathscr{Z})$ is called the primitive part of $W($ in $\mathscr{Z})$.

In [6], primitive sequences are defined in a general way. Here, to simplify the exposition, we define only open primitive sequences.

3.2. Definition. Let $X$ be a topological space. An open primitive sequence of $X$ is a sequence $\mathcal{H}$ of well-ordered open covers of $X$ such that, for each $n \in N$ :

(a) For all $H \in \mathcal{H}_{n}, p\left(H, \mathcal{H}_{n}\right) \neq \varnothing$.

(b) If $j<n$ and $p\left(H, \mathcal{H}_{n}\right) \cap p\left(H^{\prime}, \mathcal{H}_{j}\right) \neq \varnothing$, then $H \subseteq H^{\prime}$.

3.3. Definition. Let $\mathcal{H}$ be an open primitive sequence of a space $X$. A primitive representative of $\mathcal{H}$ is a sequence $H$ such that for all $n \in N$, $p\left(H_{n}, \mathcal{H}_{n}\right) \cap p\left(H_{n+1}, \mathcal{H}_{n+1}\right) \neq \varnothing$.

3.4. Notation. If $\mathcal{H}$ is a primitive sequence, $P R(\mathcal{H})$ denotes $\{H: H$ is a primitive representative of $\mathcal{H}\}$.

3.5. Definition. If $\mathcal{H}$ is a primitive sequence, then for all $H \in P R(\mathcal{H})$, $p c(H)$ denotes $\bigcap_{n \in N} p\left(H_{n}, \mathcal{H}_{n}\right)$. This set is called the primitive core of $H$.

3.6. Main theorem. A topological space is essentially $T_{1}$ and has a 
primitive base if and only if it has an open primitive sequence $\mathcal{H}$ such that for all $H \in P R(\mathcal{H})$, if $p c(H) \neq \varnothing$, then $\left\{H_{n}: n \in N\right\}$ is a base at each element of $\bigcap_{n \in N} H_{n}$.

Proof. Necessity. Let 60 be a sequence of well-ordered collections related to a space $X$ as in 2.1. Assume $X$ is essentially $T_{1}$. Call $(k, n) \in$ $N \times N$ admissible for $x \in X$ if $x$ is in $n$ elements of $C_{k}$. Let $A(k, n)=$ $\{x \in X:(k, n)$ is admissible for $x\}$. For $x \in A(k, n)$ let $W(x, i)$ denote the $i$ th element of $\mathcal{W}_{k}$ that contains $x$, let $\mathcal{W}(x, n)=\{W(x, i): i \leq n\}$, and let $V(x)$ $=\bigcap\{W(x, i): i \leq n\}$. Let $\mathcal{O}(k, n)=\{V(x): x \in A(k, n)\}$. Then for $x, y \in A(k, n)$, $V(x)=V(y)$ if and only if $\mathcal{C O}(x, n)=\mathcal{C}(y, n)$. Let $<$ be defined on $\mathcal{O}(k, n)$ by $V(x)<V(y)$ if and only if $V(x) \neq V(y)$ and if $m=\min \{i: W(x, i) \neq W(y, i)\}$, then $W(x, m)$ precedes $W(y, m)$ in $\mathcal{H}_{k}$. It is readily verified that $\leq$ well orders $\mathcal{O}(k, n)$. Furthermore $x \in p(V(x), \mathcal{O}(k, n))$ for all $x \in A(k, n)$. If $\mathcal{O}(k, n)$ covers $X$ let $\mathcal{O}^{\prime}(k, n)=\mathcal{O}(k, n)$. If not, let $\mathcal{O}^{\prime}(k, n)=\mathcal{O}(k, n) \cup\{X\}$ with an ordering which extends that of $\mathcal{O}(k, n)$ and makes $X$ follow all other elements. If $A(k, n)$ $=\varnothing$, let $\mathcal{O}^{\prime}(k, n)=\{X\}$. Let $\mathcal{U}_{(k+n-1)(k+n-2) / 2+k}$ denote $\mathcal{O}^{\prime}(k, n)$ for all $(k, n) \in N \times N$. Let $\mathcal{H}_{1}=\mathcal{U}_{1}$. Suppose $\mathfrak{H}_{1}, \ldots, \mathcal{H}_{n}$ have been defined and satisfy the conditions of 3.2 for $1 \leq i \leq n$. Call a set $W$ of $(n+1)$-form if there exist $U \in \mathcal{U}_{n+1}$ and $H \in \mathcal{H}_{n}$ such that $p\left(U, \mathcal{U}_{n+1}\right) \cap p\left(H, \mathcal{H}_{n}\right) \neq \varnothing$ and $W=U \cap H$. If $W$ is of $(n+1)$-form, then its representation is unique; i.e., if $U, U^{\prime} \in \mathcal{U}_{n+1}$ and $H, H^{\prime} \in \mathcal{H}_{n}$, and

$$
p\left(U, \mathcal{U}_{n+1}\right) \cap p\left(H, \mathcal{H}_{n}\right) \neq \varnothing \neq p\left(U^{\prime}, \mathcal{U}_{n+1}\right) \cap p\left(H^{\prime}, \mathcal{H}_{n}\right),
$$

and $U \cap H=U^{\prime} \cap H^{\prime}$, then $U=U^{\prime}$ and $H=H^{\prime}$. In what follows, if $W$ is written in $(n+1)$-form $U \cap H$, then $U \in \mathcal{U}_{n+1}$ and $H \in \mathcal{H}_{n}$. Let $\mathcal{H}_{n+1}=$ $\{W: W$ is of $(n+1)$-form $\}$ ordered by $W<W^{\prime}$ if and only if $W \neq W^{\prime}$ and if $U \cap H$ and $U^{\prime} \cap H^{\prime}$ are the respective representations of $W$ and $W^{\prime}$, then either (a) $U$ precedes $U^{\prime}$ in $\mathcal{U}_{n+1}$, or (b) $U=U^{\prime \prime}$ and $H$ precedes $H^{\prime}$ in $\mathcal{H}_{n^{\prime}}$. It may be verified that $\leq$ is a well ordering of $\mathcal{H}_{n+1}$. Furthermore, if $W \in$ $\mathcal{H}_{n+1}$ and $W=U \cap H$, then

$$
p\left(W, \mathcal{H}_{n+1}\right)=p\left(U, \mathcal{U}_{n+1}\right) \cap p\left(H, \mathcal{H}_{n}\right) .
$$

For if $x \in p\left(W, \mathcal{H}_{n+1}\right)$, then $x \in U \cap H$. If $x \in p\left(U^{\prime}, \mathcal{U}_{n+1}\right) \cap p\left(H^{\prime}, \mathcal{H}_{n}\right)$, then $W^{\prime}=U^{\prime} \cap H^{\prime} \in \mathcal{H}_{n+1}$. Since $U^{\prime} \leq U$ and $H^{\prime} \leq H$ it follows that $W^{\prime} \leq W$. Thus $W=W^{\prime}$. These considerations establish the equality. Clearly $\mathcal{H}_{n+1}$ is an open cover of $X$ since $\mathcal{H}_{n}$ is, and $p\left(H, \mathcal{H}_{n+1}\right) \neq \varnothing$ for all $H \in \mathcal{H}_{n+1}^{n+1}$. Suppose $x \in p\left(W, \mathcal{H}_{n+1}\right) \cap p\left(W^{\prime}, \mathcal{H}_{n}\right)$ and $W=U \cap H$. Then $x \in p\left(U, \mathcal{U}_{n+1}\right) \cap p\left(H, \mathcal{H}_{n}\right)$. 
Hence $H=W^{\prime}$. Thus $W \subseteq W^{\prime}$. Condition (b) of 3.3 follows from this. Thus an open primitive sequence $\mathcal{H}$ of $X$ may be defined by induction.

Suppose $H \in P R(\mathcal{H})$ and $x \in p c(H)$. Let $D$ be open and $x \in D$. Then there exist $k$ and $n$ such that $x$ is in $n$ elements of $W_{k}$ and the $n$th such element is a subset of $D$. Therefore $V(x) \in \mathcal{G}(k, n)$ and $V(x) \subseteq D$. Since $x \in p(V(x), \mathcal{O}(k, n))$ it follows for $j=(k+n-2)(k+n-1) / 2+k$ that $x \in$ $p\left(V(x), \mathcal{U}_{j}\right)$. If $j=1, V(x)=H_{1} \in \mathcal{H}_{1}$ and $V(x) \subseteq D$. If $j>1$, then there exists $H^{\prime} \in \mathcal{H}_{j-1}$ such that $x \in V(x) \cap H^{\prime} \in \mathcal{H}_{j}$, and $V(x) \cap H^{\prime} \subseteq D$. But $x \in$ $p\left(H_{j}, \mathcal{H}_{j}\right)$ implies $H_{j}=V(x) \cap H^{\prime}$. Thus $\left\{H_{n}: n \in N\right\}$ is a base at each point of $p c(H)$.

Suppose $z \in\{\bar{y}\} \cap p c(H)$. Then $y \in\{\bar{z}\}$ by essential $T_{1}$-ness. If $y \in$ $p\left(W, \mathcal{H}_{n}\right)$, then $z \in W$, so that $H_{n} \leq W$. Since $y \in H_{n}, W=H_{n}$. Thus $\{\bar{y}\} \cap$ $p c(H) \neq \varnothing$ implies $\{\bar{y}\} \subseteq p c(H)$. Suppose $z \notin p c(H)$. Then $p c(H) \subseteq X \backslash\{\bar{z}\}$. Thus $z \notin H_{j}$ for some $j \in N$. Hence $p c(H)=\bigcap_{n \in N} H_{n}$ and the proof is complete.

Sufficiency. Suppose such a sequence $\mathcal{H}$ exists in a space $X$. Then for all $x \in X$, there is $H \in P R(\mathcal{H})$ such that $x \in p c(H)$. If $D$ is open and $x \in D$, then there is a $k$ such that $H_{k} \subseteq D$. Thus $(k, 1)$ is an admissible pair for $x$. Therefore $X$ has a primitive base. Suppose $x, y \in X$ and $y \in\{\bar{x}\}$ and $x \in$ $p c(H)$. If $y \in p c(W)$, then $x \in \bigcap\left\{W_{n}: n \in N\right\}$. Thus for each $n$ there is $j>n$ such that $W_{j} \subseteq H_{n} \cap W_{n}$. Hence $y \in \bigcap\left\{H_{n}: n \in N\right.$ so that $x \in\{\bar{y}\}$. Thus $X$ is essentially $T_{1}$.

3.7. Remark. Note that the axiom of choice is not used to prove 3.6.

3.8. Example. The Sierpiński space $S=\{\varnothing, 1\}$ with topology $\{\varnothing,\{\varnothing\}, S\}$ has a primitive base but is not essentially $T_{1}$. It has exactly two primitive sequences: one has each term equal to $\{S\}$; the other is defined by $\mathcal{H}_{n}=$ $\{\{\varnothing\}, S\}$ where $\{\varnothing\}<S$. The only primitive representatives of $\mathcal{H}$ are either those whose terms are eventually $\{\varnothing\}$ or the primitive representative $H$ all of whose terms are $S$. Note that $p c(H)=\{1\}$, but $H$ is not a base at each point of $\bigcap_{n \in N} H_{n}=S$.

From 3.6 and the calculus of primitive sequences we obtain the next two theorems. Proofs will be submitted elsewhere.

3.9. Theorem. The concept of primitive base is hereditary and countably productive.

3.10. Theorem. If an essentially $T_{1}$ topological space $X$ is the union of open sets each having a primitive base in the relative topology, then $X$ has a primitive base. 
4. Spaces having bases of countable order. Theorem 4.1, whose proof may be obtained from those of Theorems 1 and 2 of [12], compares instructively with 3.6 and proves part of 2.4. Theorem 4.3 puts in sharp focus the distinction between 3.6 and 4.1 .

4.1. Theorem [12]. A topological space is essentially $T_{1}$ and has a base of countable order if and only if it has a primitive sequence $\mathcal{H}$ such that, for all $H \in P R(\mathcal{H})$, if $\bigcap_{n \in N} H_{n} \neq \varnothing$, then $\left\{H_{n}: n \in N\right\}$ is a base at each point of $\bigcap_{n \in N} H_{n}$.

4.2. Definition. Suppose $X$ is a topological space and $\mathcal{H}$ is an open primitive sequence of $X$ satisfying the conditions of (3.6). Then $\mathcal{H}$ will be called a primitive sequence (of $X$ ) of basic type.

4.3. Theorem. A topological space is essentially $T_{1}$ and has a base of countable order if and only if it has a primitive sequence $\mathcal{H}$ of basic type such that for all $H \in P R(\mathcal{H})$, if $\bigcap_{n \in N} H_{n} \neq \varnothing$, then $p c(H) \neq \varnothing$.

Proof. Necessity. Suppose $X$ is essentially $T_{1}$ and has a base of countable order. Let $\mathcal{H}$ be a sequence relative to $X$ as in 4.1 , and let $H \in$ $P R(\mathcal{H})$ and $x \in \bigcap_{n \in N} H_{n}$. There exists $W \in P R(\mathcal{H})$ such that $x \in p c(W)$. Thus each $W_{n} \leq H_{n}$. Since $\left\{H_{n}: n \in N\right\}$ is a base at $x$, there exists $j>n$ such that $H_{j} \subseteq H_{n} \cap W_{n}$. Since $p\left(H_{j}, \mathcal{H}_{j}\right) \subseteq p\left(H_{n}, \mathcal{H}_{n}\right)$, it follows that $H_{n} \leq$ $W_{n^{*}}$ Thus $p c(H)=\bigcap_{n \in N} H_{n^{*}}$

4.4. Remark. This makes precise a sense in which essentially $T_{1}$ spaces having bases of countable order are complete spaces in the class of spaces having primitive bases.

5. Completeness. A natural question arises concerning completeness. We discuss it for the regular $T_{0}$ case although an analogous discussion can be given for pararegular [7] spaces. Recall that a regular $T_{0}$ space having a base of countable order is called an Aronszajn space [6] and a monotonically complete member of this class is called a complete Aronszajn space. Does there exist for the class of regular $T_{0}$ spaces having a primitive sequence of basic type a "natural" class of complete spaces? Theorem 5.3 shows that a natural method of defining a complete space in this case (one analogous to that used for Aronszajn spaces) yields the complete Aronszajn spaces rather than a new class. Theorems 5.1 and 5.2 imply 5.3.

5.1. Theorem. Suppose $X$ is a topological space having a primitive sequence $\mathcal{H}$ of basic type. Then the following are equivalent: 
(a) $H \in P R(\mathcal{H})$ implies $\bigcap_{n \in N} H_{n} \neq \varnothing$ and $\left\{H_{n}: n \in N\right\}$ is a base at each point of $\bigcap_{n \in N} H_{n}$.

(b) $H \in P R(\mathcal{H})$ implies $p c(H) \neq \varnothing$.

Proof. Clearly (b) implies (a). The proof of 4.3 shows that (a) implies (b).

5.2. Theorem. A topological space is a complete Aronszaja space if and only if it is regular $T_{0}$ and has an open primitive sequence $\mathcal{H}$ such that for all $H \in P R(\mathcal{H}), \bigcap_{n \in N} H_{n} \neq \varnothing$ and $\left\{H_{n}: n \in N\right\}$ is a base at each point of $\bigcap_{n \in N} H_{n}$

Proof. A proof for this may be obtained from the proofs of Theorems 3.2, 3.3, and Lemma 2.4 of [6].

5.3. Theorem. A topological space is a complete Aronszajn space if and only if it is a regular $T_{0}$ space having a primitive sequence $\mathcal{H}$ of basic type such that for all $H \in P R(\mathcal{H}), p c(H) \neq \varnothing$.

Added in proof. (1) A proof of Theorem 2.6 is to appear in a paper by the authors in the Canadian Journal of Mathematics in 1975. (2) The proof of Theorem 3.6 shows that essentially $T_{1}$ may be omitted in its statement if $\bigcap_{n \in N} H_{n}$ is replaced by $p c(H)$. (Dr. T. M. Phillips proved a similar theorem in his 1974 University of Oklahoma dissertation.)

\section{REFERENCES}

1. H. R. Bennett, On quasi-developable spaces, General Topology and Appl. 1 (1971), 253-262. MR 44 \#5921.

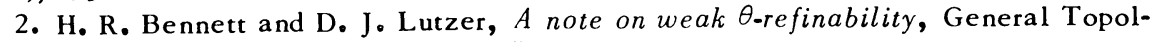
ogy and Appl. 2 (1972), 49-54. MR $46 \# 853$.

3. A. S. Davis, Indexed systems of neighborhoods for general topological spaces, Amer. Math. Monthly 68 (1961), 886-893. MR 35 \#4869.

4. E. Michael, The product of a normal space and a metric space need not be normal, Bull. Amer. Math. Soc. 69 (1963), 375-376. MR 27 \#2956.

5. H. H. Wicke, Base of countable order theory and some generalizations, Proc. Univ. Houston Point Set Topology Conf. 1971, Houston, Tex., 1972.

6. H. H. Wicke and J. M. Worrell, Jr., Topological completeness of first countable Hausdorff spaces. I, Fund. Math. 75 (1972), 209-222. MR $46 \# 8189$.

7. - Pararegularity, Notices Amer. Math. Soc. 20 (1973), A-173. Abstract \#701-54-15.

8. - Characterization of absolute sets of interior condensation, Notices Amer. Math. Soc. 20 (1973), A-401. Abstract \#704-G6.

9. - The preservation of primitive base by certain open mappings, Notices Amer. Math. Soc. 20 (1973), A-533. Abstract \#706-54-5.

10. - Uniformly primitively complete mappings, Notices Amer. Math. Soc. 20 (1973), A-675. Abstract \#709-G23.

11. J. M. Worrell, Jr. and H. H. Wicke, Concerning spaces having bases of countable order, Notices Amer. Math. Soc. 12 (1965), 343. Abstract \#622-60. 
12. J. M. Worrell, Jr. and H. H. Wicke, Characterizations of developable topological spaces, Canad. J. Math. 17 (1965), 820-830. MR 32 \#427.

13. - A central metrization theorem. I, Notices Amer. Math. Soc. 20 (1973), A-355. Abstract \#702-G2.

14. J. M. Worrell, Jr. and H. H. Wicke, A central metrization theorem. II, Notices Amer. Math. Soc. 20 (1973), A-381. Abstract \#703-G14.

15. L. Zippin, On a problem of N. Aronszajn and an axiom of R. L. Moore, Bull. Amer. Math. Soc. 37 (1931), 276-280.

DEPARTMENT OF MATHEMATICS, OHIO UNIVERSITY, ATHENS, OHIO 45701 